\title{
BIBECHANA
}

A Multidisciplinary Journal of Science, Technology and Mathematics ISSN 2091-0762 (Print), 2382-5340 (Online)

Journal homepage: http://nepjol.info/index.php/BIBECHANA

Publisher: Research Council of Science and Technology, Biratnagar, Nepal

\section{Far infrared cavity of a post C-rich AGB star under IRAS survey}

\author{
A.K. Gautam*, B. Aryal \\ Central Department of Physics, T.U., Kirtipur,Nepal \\ "Email: arjungautamnpj@gmail.com \\ Article history: Received 26 October, 2017; Accepted 03 December, 2017 \\ DOI: http://dx.doi.org/10.3126/bibechana.v15i0.18506
}

This work is licensed under the Creative Commons CC BY-NC License. https://creativecommons.org/licenses/bync/4.0/

\begin{abstract}
In this paper, we discuss about the physical properties of the dusty environment around the mass losing

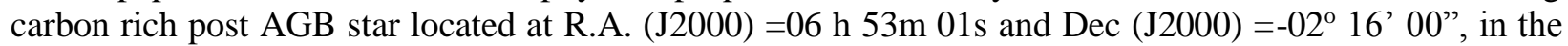
far infrared IRAS maps. A cavity like structure (major diameter $\sim 103.3 \mathrm{pc} \&$ minor diameter $\sim 33.1 \mathrm{pc}$ ) is found to lie at R.A. $(\mathrm{J} 2000)=06 \mathrm{~h} 51 \mathrm{~m} 54.02 \mathrm{~s}$ and DEC $(\mathrm{J} 2000)=-01^{\circ} 35^{\prime}$, 43", located at a distance $\sim 6.11 \mathrm{kpc}$ from the star. We studied the distribution of flux density, dust color temperature, dust mass in the cavity. The dust color temperature is found to lie in the range $18.7 \mathrm{~K}$ to $20.5 \mathrm{~K}$ which shows the cavity is isolated and independently evolved. Such a low offset temperature variation shows that there is symmetric outflow or symmetric distribution of density and temperature. It further suggests that our structure is bigger in size and is far away from the far infrared loops(KK loops). The cavity may be in thermally pulsating phase. A possible explanation of the results will be discussed.
\end{abstract}

Keywords: AGB stars; Post AGB Stars; Dust color temperature; Dust mass.

\section{Introduction}

Asymptotic giant branch (AGB) stars are the final nuclear burning stage of low- and intermediate-mass stars driven by nuclear burning. This phase of evolution is characterized by two nuclear burning shell of hydrogen and helium where hydrogen burning shell lies below the convective envelope and helium burning shell lies above the electron-degenerate core of carbon and oxygen, or for the most massive AGB stars a core of oxygen, neon, and magnesium [1]. This AGB stage is characterized by low surface

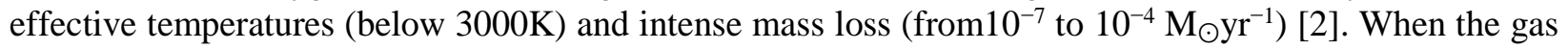
temperature drops to the sublimation temperature range, heavy elements in the mass outflow from a central star will condense to form dust. Dusty circumstellar envelopes will format the distance of several stellar radii. Dust grains in the envelopes absorb stellar radiation and re-emit infrared radiation so AGB stars are important infrared sources. The mass loss process plays an important role in the evolution of AGB stars . It affects the lifetime of the AGB phase and the core-mass of the subsequent post-AGB stars. Statistics of a large sample of AGB stars would help to constrain the evolution of dust envelope. 
There are two main types of AGB stars: the O-rich with $\mathrm{C} / \mathrm{O}<1$ and mainly silicate-type grains in the outflow, and C-rich with $\mathrm{C} / \mathrm{O}>1$ and mainly carbonaceous grains in the envelopes. Due to different dust compositions of these two types of AGB stars, different infrared spectral features are obtained which can be used to distinguish the two groups of the stellar objects. Most of the carbon compounds such as aromatic hydrocarbon, benzene, methane, etc. are responsible for biological life so carbon- rich AGB stars are preferred in our research work.

He-core burning phase is about 10 times shorter than the H-core burning shell so that the He-core burning leaves a $\mathrm{C} / \mathrm{O}$ core behind that is surrounded by both a hydrogen and helium burning shell. For low and intermediate mass stars, carbon doesn't ignite and $\mathrm{C} / \mathrm{O}$ core contracts and becomes electron degenerate. During the early AGB phase, the abundance of $\mathrm{He}$ in the centre goes to zero where He-burning continues in a shell around a degenerate $\mathrm{C}-\mathrm{O}$ core. In the meantime, the $\mathrm{H}$ - layer around the helium shell expands and cools sufficiently so that hydrogen burning shell is extinguished. Convective envelope sets in and moves inwards and second dredge-up takes place. He shell is the main source for nuclear production so that it burns outward and reaches the hydrogen shell. In case of thermally pulsating AGB phase, helium shell becomes thin and remains thermally unstable as a result thermal pulses are produced. In each thermal pulse, luminosity of helium shell nearly approaches $10^{8} \mathrm{~L} \odot$ [3]. The production of such high luminosity in helium shell is called He shell flash or thermal pulse which is used to expand the outer layers. Such strong expansion drives the $\mathrm{H}$ shell cooler and less dense as a result $\mathrm{H}$ shell is extinguished. The inner edge of deep convective envelope can then move inward and mix to the surface products of internal nucleosynthesis. This mixing process which occurs periodically after each TP is known as third dredge-up which is the mechanism for producing carbon stars. During TP-AGB phase, main dominant source of nuclear energy is the hydrogen shell. Thermally pulsating AGB phase is the phase after the first thermal pulse to the time when the star ejects its envelope.

At the end of the TP-AGB, the envelope mass is strongly reduced down to $0.05 \mathrm{M}_{\odot}$ due to the strong mass loss. The star now evolves towards high temperatures at an almost constant luminosity. This is because the surface layer is gradually heating up due to the proximity of the stable burning thin H-shell which produces the luminosity. The star has now entered the post-asymptotic giant branch (post-AGB) phase.

The material expelled away from the system during the AGB stage forms a slowly expanding circumstellar shell [4]. When the mass of the hydrogen-rich envelope drops to $\approx 10^{-3} \mathrm{M}_{\odot}$, it starts to contract at a constant luminosity. This contraction phase causes an increase in effective temperature and at the same time leads to a radiatively driven wind which can compress the circumstellar shell and may result in ionization of the circumstellar material. In other words, the star may become hot enough to ionize its circumstellar material, observable as a planetary nebula (PN), in a few hundred years [5]. The star at this stage is known as a post-AGB star. These have luminosity classes ranging from I (supergiant) to III (giant) with spectral types from B to K. Typical post-AGB stars are expected to have luminosities around $10^{3}-10^{4} \mathrm{~L}_{\odot}[6]$. Masses of these objects are in the range $0.6 \mathrm{M}_{\odot}-1 \mathrm{M}_{\odot}$. The star leaves the AGB with $\mathrm{T}_{\text {eff }}<5000 \mathrm{~K}$. When it reaches $\mathrm{T}_{\text {eff }}>30,000$, it may ionize the remnant nebula.

In this paper, we study the physical properties of a far infrared cavity, that we investigated during a systematic search on IRAS maps, located close to a carbon-rich post AGB star (PAGB 06-02) at -1.3 latitude. In section 2 , we describe methods of calculation. A brief description of the result and discussion will be given in the section 3. Finally, we conclude our results in the section 4.

\section{Methods}

We investigated a cavity-like structure in both 60 and 100 micron IRAS maps around a PAGB star. Fig.1(a), and 1(b) are cavity images at 60 and $100 \mu \mathrm{m}$ whereas 1(c) is its contour map. We briefly describe a method for calculation of dust color temperature and dust mass of the dusty environment around carbon-rich post Asymptotic Giant Branch named PAGB06-02. 


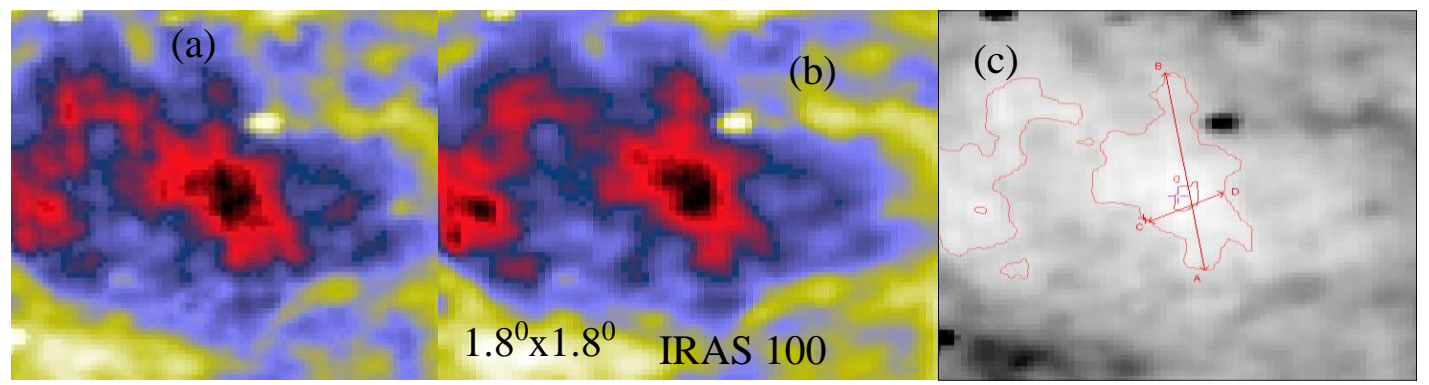

Fig. 1: (a) and (b) IRAS $60 \mu \mathrm{m}$ and $100 \mu \mathrm{m}$ far infrared image of the core region of AGB 06-02 centered at R.A.

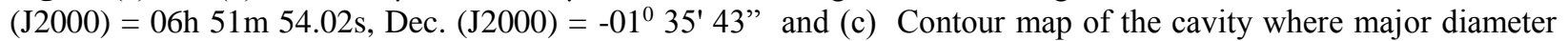
$(\mathrm{AB})$ and minor diameter $(\mathrm{CD})$ passing through minimum flux.

\subsection{Dust Color Temperature Estimation}

By using the IRAS $60 \mu \mathrm{m}$ and $100 \mu \mathrm{m}$ flux densities, Schnee et al.[7] calculated dust color temperature. The flux density of emission at a wavelength $\lambda_{\mathrm{i}}$ is given by

$$
\mathrm{F}_{\mathrm{i}}=\left[\frac{2 h c}{\lambda_{i}^{3}\left(e^{\frac{h c}{\lambda_{i} k T_{d}}}\right)-1}\right] \mathrm{N}_{\mathrm{d}} \alpha \lambda_{\mathrm{i}}^{-\beta} \Omega_{i}
$$

where $\beta$ is the spectral emissivity index, $N_{d}$ is the column density of dust grains, $\alpha$ is a constant i.e. free parameter which relates the flux with the optical depth of the dust, and $\Omega_{\mathrm{i}}$ is the solid angle subtended at $\lambda_{\mathrm{i}}$ by the detector. In Dupac et al. [8], there is an inverse relationship between temperature and emissivity spectral index. We have with the assumptions that the dust emission is optically thin at $60 \mu \mathrm{m}$ and 100 $\mu \mathrm{m}$ and that $\Omega \omega \sim \Omega 100$ (true for IRAS image), we can write the ratio, R, of the flux densities at $60 \mu \mathrm{m}$ and $100 \mu \mathrm{m}$ as

$$
\mathrm{R}=0.6^{-(3+\beta)} \frac{e^{144 / T_{d}}-1}{e^{240 / T_{d}}-1}
$$

The spectral emissivity index $(\beta)$ depends on dust grain properties like composition, size, and compactness. For a pure blackbody would have $\beta=0$, the amorphous layer-lattice matter has $\beta \sim 1$, and the metals and crystalline dielectrics have $\beta \sim 2$ which is used in our calculations. For a smaller value of $\mathrm{T}_{\mathrm{d}}, 1$ can be dropped from both numerator and denominator of Eq. (2) and it takes the form

$$
\mathrm{R}==0.6^{-(3+\beta)} \frac{e^{144 / T_{d}}}{e^{240 / T_{d}}}
$$

where, $R=\frac{F(60 \mu \mathrm{m})}{F(100 \mu \mathrm{m})}$

Taking natural logarithm on both sides of Eq. (3) and solving it, we find the expression for the temperature as

$$
\begin{aligned}
\ln (\mathrm{R}) & =\ln 0.6^{-(3+\beta)}\left[144 / \mathrm{T}_{\mathrm{d}}-240 / \mathrm{T}_{\mathrm{d}}\right] \\
& =\ln 0.6^{-(3+\beta)}\left[-96 / \mathrm{T}_{\mathrm{d}}\right] \\
\mathrm{T}_{\mathrm{d}}= & \frac{-96}{\ln \left\{\mathrm{R} \times 0.6^{(3+\beta)}\right\}}
\end{aligned}
$$


$\mathrm{F}(60 \mu \mathrm{m})$ and $\mathrm{F}(100 \mu \mathrm{m})$ are the flux densities in $60 \mu \mathrm{m}$ and $100 \mu \mathrm{m}$ respectively and Eq. (5) can be used for calculation of the dust grain temperature.

\subsection{Dust Mass Estimation}

Dust mass is another important physical quantity which is useful to analysis the cavity structure. We need the known distance of the loops to calculate its dust mass which was provided in catalog of far infrared loops in the galaxy [9]. For the calculation of dust mass, we first obtained the value of flux density $\left(S_{v}\right)$ at $100 \mu \mathrm{m}$ maps. The dust mass is estimated using [10],

$$
\mathrm{M}_{\text {dust }}=\frac{4 a \rho}{3 Q_{n}}\left|\frac{\mathrm{S}_{\mathrm{n}} \mathrm{D}^{2}}{\mathrm{~B}(\mathrm{n}, \mathrm{T})}\right|
$$

where, weighted grain size $(\mathrm{a})=0.1 \mu \mathrm{m}$, grain density $(\rho)=3000 \mathrm{~kg} \mathrm{~m}^{-3}$, grain emissivity $\left(\mathrm{Q}_{\mathrm{v}}\right)=0.0010$ (for $100 \mu \mathrm{m}$ ) [11].

The Planck's function $\mathrm{B}(v, T)$, which is the function of temperature and frequency and is given by the expression:

$$
\mathrm{B}(v, T)=\frac{2 h c}{\lambda^{3}}\left(\frac{1}{\frac{\mathrm{hc}}{\mathrm{e}^{\mathrm{lkT}}-1}}\right)
$$

where, $\mathrm{h}=$ Planck's constant, $\mathrm{c}=$ velocity of light, $v=$ frequency at which the emission is observed, $\mathrm{T}=$ the average temperature of the region.

For $100 \mu \mathrm{m}$ wavelength, the expression for the dust mass (5) reduces to,

$$
\mathrm{M}_{\text {dust }}=0.4\left[\frac{S_{\vartheta} D^{2}}{B(\vartheta, T)}\right]
$$

We use equation (7) to calculate dust mass of the cavity.

\section{Result and Discussion}

\subsection{Structure: Contour Maps}

While going through the systematic search on IRAS maps, we discovered an isolated cavity in the 100 $\mu \mathrm{m}$ and $60 \mu \mathrm{m}$ at R.A. (J2000) $=06^{\mathrm{h}} 51^{\mathrm{m}} 54.02^{\mathrm{s}}$ and Dec. (J2000) $=-01^{\mathrm{o}} 35^{\prime} 43^{\prime}$. With the help of the software ALADIN2.5, we have studied physical properties (size, dust color temperature, dust mass, etc) of the cavity. We selected contour level in such a way that it circles the cavity. The major axis, minor axis and line passing through minimum temperature and minimum flux are shown in the Fig.1(b).

\subsection{Flux Density and its Variation}

By using ALADIN 2.5 software, the values of flux densities at $60 \mu \mathrm{m}$ and $100 \mu \mathrm{m}$ have measured. The flux density distribution within the contour of the region of interest has studied. We plotted a graph between flux at $100 \mu \mathrm{m}$ and $60 \mu \mathrm{m}$ with the help of ORIGIN 5.0 which is shown in Fig.2(a). From the linear fit, slope of the line was 0.25 . The linear equation of the fitted line is, $y=-2.87+0.25 x$. Using the slope of best fitted plot, dust color temperature is found as $24.4 \mathrm{~K}$ which is slightly more than our calculated value.

Again distribution of flux at $100 \mu \mathrm{m}$ of the pixels within the contour level with right ascension (R.A.) and declination (Dec.) are plotted by using ORIGIN 8.0 and the graph is shown in Fig.2(b). Graph shows that all the fluxes from minimum to maximum lie within the contour level. Most of the maximum flux regions lie at the boundary.

\subsection{Dust color Temperature and its Variation}

Using the method of [7], we calculated dust color temperature of each pixel inner the outer isocontour in the region of interest. We use the IRAS $100 \mu \mathrm{m}$ and $60 \mu \mathrm{m}$ FITS images downloaded from the IRAS 
server. For the calculation of temperature we choose the value of $\beta=2$ following the explanation given by [8]. Variation of temperature with corresponding R.A.(J2000) and Dec.(J2000) are plotted by using ORIGIN 8.0 and the graph is shown in Fig. 3(a). Graph shows that temperature distributions are in separate cluster but minimum temperature region is little bit shifted from minimum flux density which is unusual behaviour. Such type of nature is obtained due to external factors.
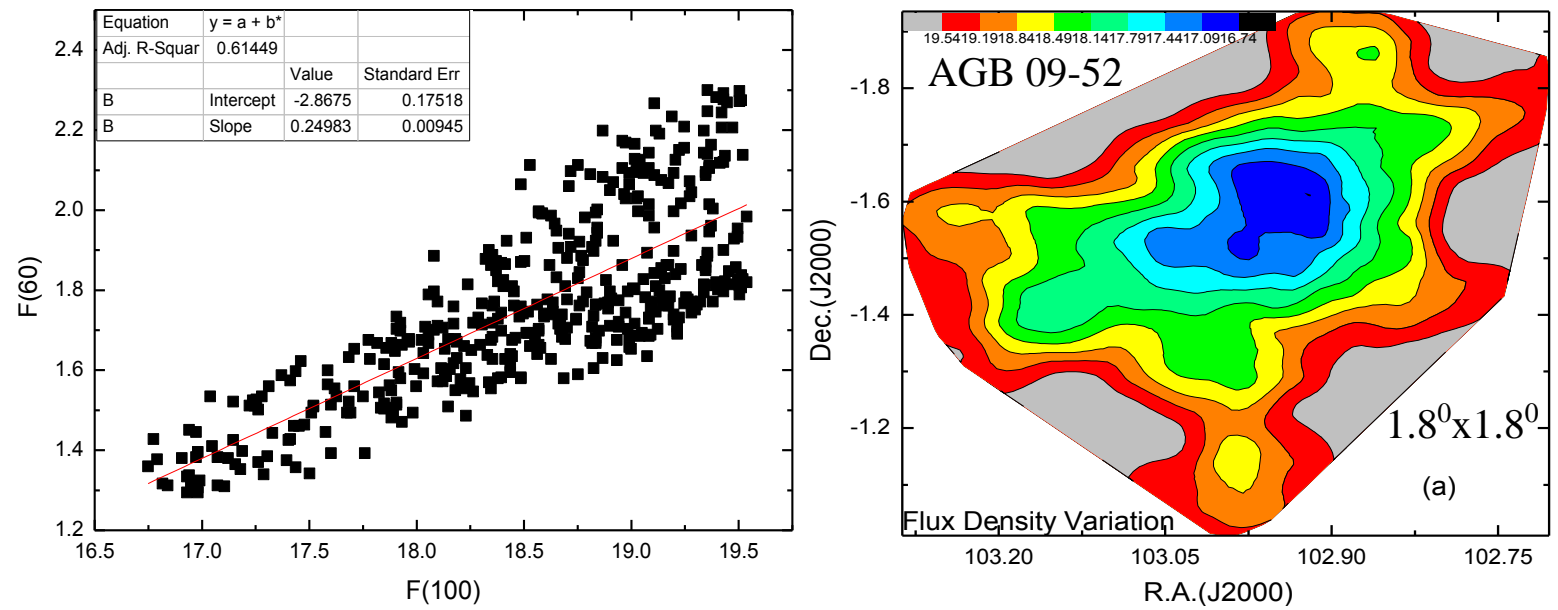

Fig.2: (a) The $100 \mu \mathrm{m}$ verses $60 \mu \mathrm{m}$ flux density in the region of interest and 2(b) Contour map at $100 \mu \mathrm{m}$ flux density where the AGB star is located at the center R.A. $(\mathrm{J} 2000)=06^{\mathrm{h}} 51^{\mathrm{m}} 54.02^{\mathrm{s}}$, Dec. $(\mathrm{J} 2000)=-01^{0} 35^{\prime} 43^{\prime \prime}$.
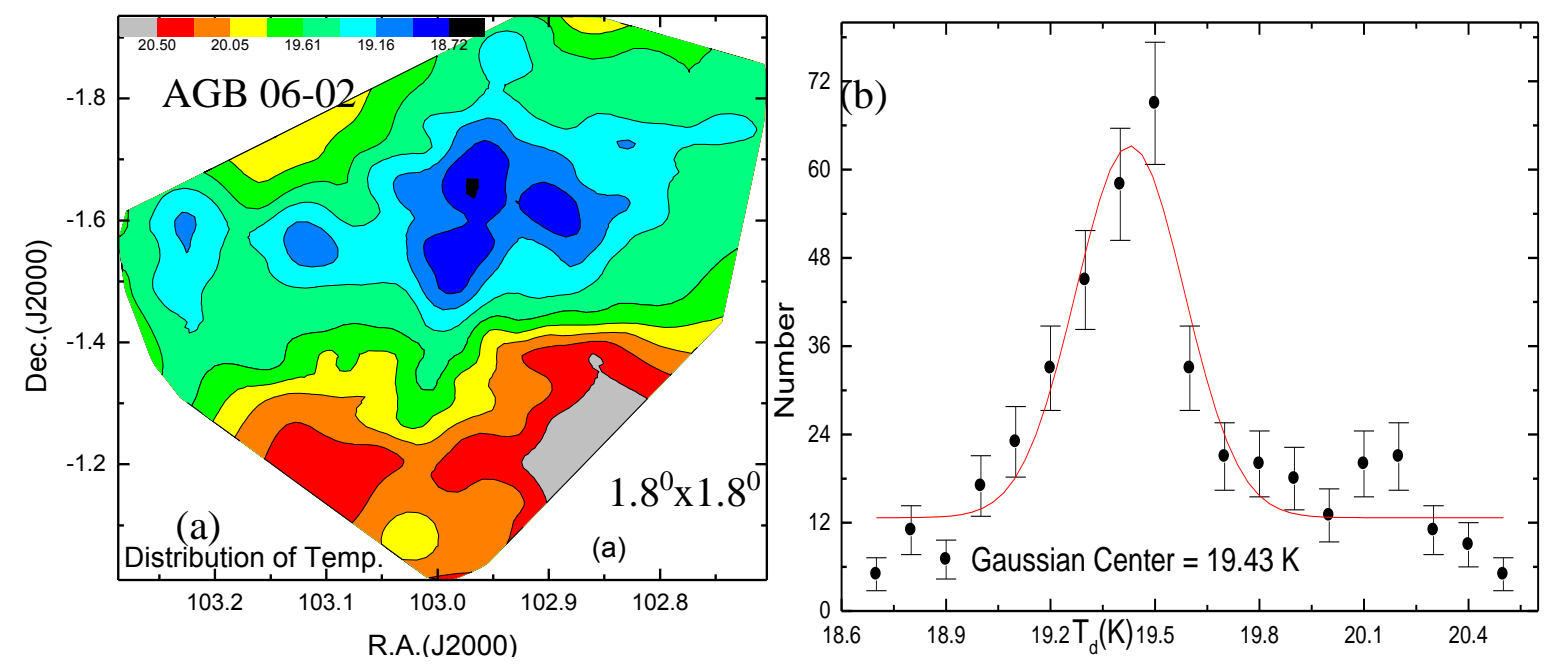

Fig. 3: (a) Contour map of dust color temperature and (b) Gaussian fit between dust color temperature and number of pixels. The field is centered at R.A. $(\mathrm{J} 2000)=06^{\mathrm{h}} 51^{\mathrm{m}} 54.02^{\mathrm{s}}$ and Dec. $(\mathrm{J} 2000)=-01^{\circ} 35^{\prime} 43^{\prime \prime}$.

The region in which minimum and maximum temperature is found in the range of $18.7 \mathrm{~K}$ to $20.5 \mathrm{~K}$ with an offset temperature of dust $1.8 \mathrm{~K}$. Such a low offset temperature variation shows that there is symmetric outflow or symmetric distribution of density and temperature. It further suggests that particles are independently vibrating. The cavity may be in thermally pulsating phase. When this result is compared with the result obtained in [12] where temperature variation is $20 \mathrm{~K}$ to $22 \mathrm{~K}$ so our result is also 
comparable with that result. In the contour map, minimum flux and minimum temperature region are shifted which is due to some external factors possibly due to AGB wind. There is no good agreement in case of temperature in the Gaussian fit (Fig. 3b) with offset $12.69 \mathrm{~K}$.

\subsection{Size of the Structure}

Major and minor diameter of the structure can be easily calculated by using a simple expression i.e., $\mathrm{L}=$ $\mathrm{R} \times \theta$, where $\mathrm{R}=6.11 \mathrm{kpc}$ is the distance of the structure [9 ] and $\theta=$ pixel size (in radian). After calculation the major and minor diameter of the cavity region are found to be $103.3 \mathrm{pc}$ and $33.1 \mathrm{pc}$ respectively. Thus, the size of the structure is $103.3 \mathrm{pc} \times 33.1 \mathrm{pc}$.

\subsection{Dust Mass Estimation and its variation}

For the calculation of dust mass, we need the distance to the region of interest. The distance of the structure is $6.11 \mathrm{kpc}$ [9]. By using the temperature of each pixel and corresponding distance of the structure, we calculated mass of each pixel. Average mass of each pixel is $5.6 \times 10^{28} \mathrm{~kg}$ and total mass of the structure is $2.5 \times 10^{31} \mathrm{~kg}$ i.e $12.6 \mathrm{M} \odot$. But mass of dust obtained around white dwarf WD 1003-44 in $[12,5]$ is $0.08 \mathrm{M} \odot$. It means mass of dust around AGB Star is less than White Dwarf.
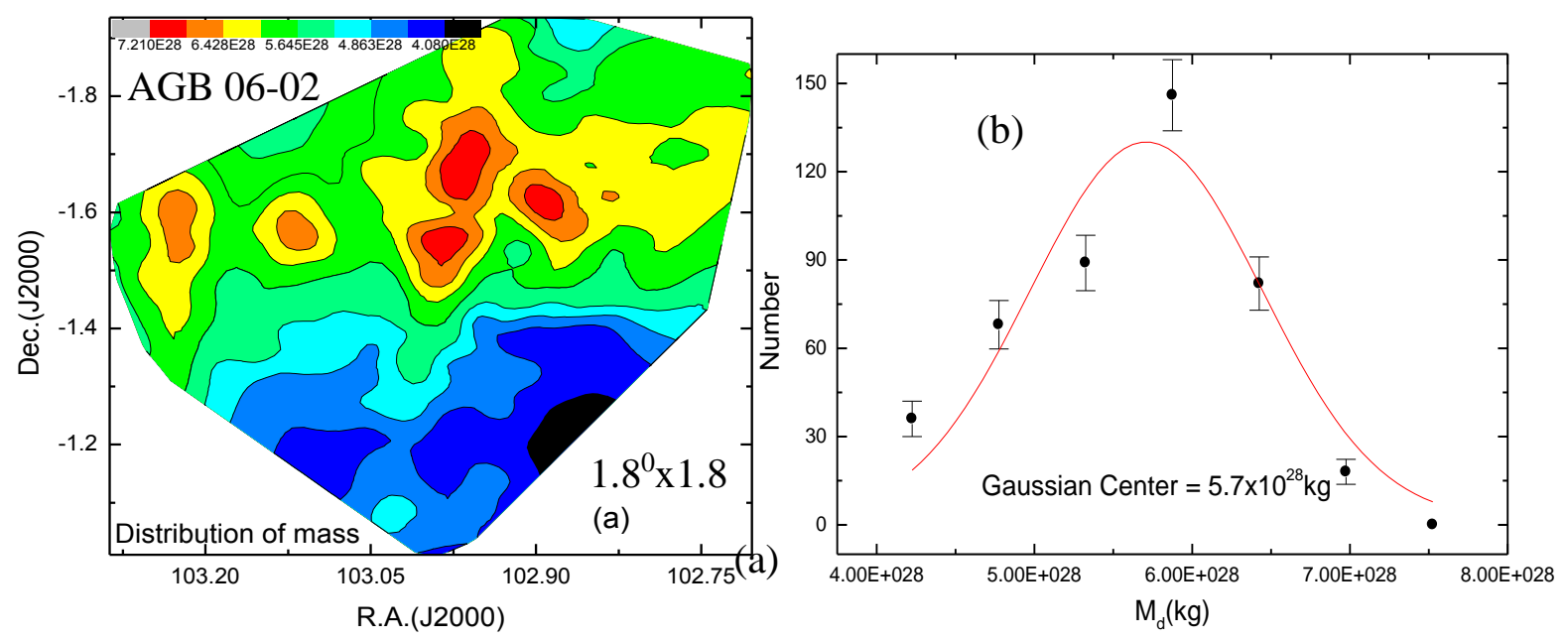

Fig.4: (a) contour map of dust mass and (b)Gaussian fit between mass and number of pixels. The field is centered at R.A. $(\mathrm{J} 2000)=06^{\mathrm{h}} 51^{\mathrm{m}} 54.02^{\mathrm{s}}$ and Dec. $(\mathrm{J} 2000)=-01^{0} 35^{\prime} 43^{\prime \prime}$.

Distribution of dust mass of the pixels within the selected contour level with R.A. (J2000) and Dec.(J2000) are plotted in contour map by using ORIGIN 8.0. Graph obtained is shown in Fig.4(a) which shows that minimum mass region didn't lie at the maximum temperature region in the selected contour which is unusual trend and is possibly due to AGB wind. There is around good agreement in case of dust mass where offset mass is $1.6 \mathrm{~kg}$. Graph $4(\mathrm{~b})$ is the Gaussian fit between mass and number of pixels where offset mass is $1.6 \mathrm{~kg}$.

\section{Conclusion}

The physical properties of the cavity-like structure that we investigated while searching an effect of AGB wind around carbon-rich AGB stars. A study of flux density and dust color temperature maps mass of dust was calculated. Our conclusions are as follows:

- The major and minor diameter of the cavity like structure was found to be $103.3 \mathrm{pc}$ and $33.1 \mathrm{pc}$ respectively. 
- The maximum temperature $20.5 \mathrm{~K}$ was found at R.A.(J2000) $=102.85^{\circ} \&$ Dec. $(\mathrm{J} 2000)=-1.36^{0}$ and minimum temperature $18.7 \mathrm{~K}$ was found at R.A.(J2000) $=102.98^{\circ} \&$ Dec.(J2000) $=-1.55^{0}$ with offset of $1.8 \mathrm{~K}$. The small value of dust color temperature in the cavity suggests a continuous process by which cavity is supposed to be formed. Low offset in the temperature hints that there is symmetric outflow or symmetric distribution of density and temperature.

- In general, minimum flux and minimum temperature lie at same point in the pixel and in this case nearly normal condition is achieved. Similarly maximum temperature and minimum mass region lie nearly at same region which is normal behavior.

- Average mass of each pixel is $5.6 \times 10^{28} \mathrm{~kg}$ and total mass of the cavity is $2.5 \times 10^{31} \mathrm{~kg}$.

We intend to study the role of cabon-rich PAGB star to form the far-infrared cavity in the future.

\section{Acknowledgements}

We are grateful to the Department of Astro-Particle Physics, Innsbrck University, specially to Prof. R. Weinberger for invoking us to work on dusty environments around AGB stars. This research has made use of SkyView Virtual Observatory, Aladin v2.5 and NASA/IPAC Extragalactic Database (NED). One of the authors (AKG) acknowledges Central Department of Physics, T.U., Nepal for providing various support of Ph.D.

\section{References}

[1] F. Herwig, Evolution of Asymptotic Giant Branch Stars, ARAA, 43 ( 2005) 435. doi.org/10.1146/annurev.astro.43.072103.150600.

[2] Kyung-Won Suh, Astrophysics of dusty stellar winds from AGB stars, Journal of the Korean astrophysical Society 47(2014) 219-233. doi.org/10.5303/JKAS.2014.47.6.219.

[3] A.I. Karakas, J.C. Lattanzio and O.R. Pols, Parameterising the third dredge- up in asymptotic giant branch stars, Astron. Soc. Aust. 19 ( 200 ) 515.

[4] Jr. Icko Iben and A.Renzini, Asymptotic giant branch evolution and beyond, Annual review of Astronomy and Astrophysics 21(1983) 271.

[5] R.D. Oudmaizer, A search for hot- post AGB stars in the IRAS point source catalog, Astronomy and Astrophysics 306 (1996) 823.

[6] T. Blocker, Stellar evolution of low-and intermediate mass stars II. Post AGB evolution, Astronomy and Astrophysics 299 (1995) 755.

[7] S.L. Schnee, N. A. Ridge, A.A. Goodman, G. L. Jason, A Complete Look at the Use of IRAS Emission Maps to Estimate Extinction and Dust Temperature, APJ .634 (2005) 442. doi.org/10.1086/511054.

[8] X. Dupac, J.P. Bernard, N. Boudet, M. Giard, J.M. Lamarre, C. Meny, F. Pajot, I. Ristorcelli, G. Serra, B. Stepnik, J.P. Torre,Inverse Temperature Dependance of the Dust Submillimeter Spectral Index, A\&A, 404 (2003) L11. doi.org/10.1051/0004-6361:200030575.

[9] R. Szczerba, N. Siodmiak, G. Stasinska and J.Borkowski, An evolutionary catalogue of galactic post-AGB and related objects, Astronomy and astrophysics 469 (2007) 799.

doi.org / 10.1051/0004-6361:20067035.

[10] R.H. Hildebrand, The determination of cloud mass and dust characteristics from sub millimeter thermal emission, Q.Jl.R.astr. obs.ser 24 (1983) 267.

[11] K. Young, T.G. Phillips, G.R. Knapp, Circumstellar Shells Resolved in IRAS Survey Data II. Analysis, ApJ, 409 (1993) 725.

[12] B. Aryal, R. Weinbergerr., Dust structure around White Dwarf WD 1003-44 in 60 and $100 \mu \mathrm{m}$ Iras Survey". The Himalayan Physics II (2011) 5. 